\title{
Datos sobre la historia del español en Centroamérica: el nivel fónico en la Guatemala del siglo XVIII
}

\section{Some Data on the History of Central American Spanish: the Phonic Level in the 18th Century Guatemala}

José LuIs RAmírez Luengo [jose_ramirez14@hotmail.com]

Universidad Autónoma de Querétaro, México

\begin{abstract}
RESUMEN
Dada la escasez de estudios sobre la diacronía del español guatemalteco, este trabajo pretende contribuir al conocimiento histórico de esta variedad en los siglos XVII y XVIII por medio del estudio de su configuración fónica: se analizará, así, un corpus de documentos inquisitoriales (Ramírez Luengo 2017) y, por medio de la valoración de las grafías que rompen las tendencias gráficas de la época, se analizarán todos los fenómenos fonéticos y fonológicos que los textos reflejan, intentando ofrecer no solo una descripción coherente de estos en relación con la situación de la Guatemala actual (Lipski 2000; Utgård 2010), sino también una interpretación adecuada a la luz de los principios de la dialectología histórica, de modo que se pueda comprobar si en esta centuria se descubre una configuración fónica semejante a la que existe hoy y, por tanto, si se ha producido ya en estos momentos la dialectalización de este nivel lingüístico.
\end{abstract}

\section{Palabras clave}

Historia del español de América; Guatemala; fonética-fonología; siglo XVIII; documentación de archivo

\begin{abstract}
Since diachronic studies of Guatemalan Spanish are scarce, this paper intends to add on the historic understanding of 17th and 18th centuries in such a variety of Spanish, through the study of its phonic set-up. A corpus of inquisitorial documents (Ramírez Luengo 2017) shall be analyzed, as well as every phonetic and phonological phenomena mirrored in a corpus of inquisitorial documents (Ramírez Luengo 2017) shall be analyzed, by assessing the value of trend-breaking spelling at the time. This research is meant to provide not only a coherent description of such phenomena in relation to present day situation in Guatemala (Lipski 2000; Utgård 2010), but also an appropriate interpretation under the principles of historical dialectology. The end goal is to determine the existence of a phonic set-up in that century that would be analogue to present day's setting, and thus confirm the dialectalization on this linguistic level in this period.
\end{abstract}

\section{KEYWORDS}

History of Latin American Spanish; Guatemala; phonetics-phonology; 18th century; archive documentation

RECIBIDO 2018-03-15; ACEPTADO 2018-05-21 
1. Aunque afortunadamente Guatemala parece estar dejando de ser ese hueco en blanco que hasta el momento actual ha representado dentro de los estudios sobre la historia del español de América, no cabe duda de que es aún mucho lo que se debe investigar al respecto si lo que se pretende es ofrecer una visión más o menos coherente de los procesos diacrónicos que han dado como resultado la variedad lingüística que se emplea hoy en el país: en efecto, es importante señalar que se cuenta ya con algunos análisis sobre determinados aspectos fónicos, morfosintácticos y léxicos del español guatemalteco de los siglos XVIII y XIX (Hernández 2008a, 2008b; Pérez Cordón y Ramírez Luengo 2007; Polo Cano 2005; Ramírez Luengo 2003, 2006; Ramírez Luengo, Aquino Melchor y Ramírez Vázquez 2017; Taracena Arriola 1985), pero no cabe duda de que tales análisis resultan todavía muy parciales, de manera que se hace difícil obtener a partir de ellos una visión clara del perfil dialectal que identifica a dicha variedad regional en los diferentes momentos de su historia. Así las cosas, parece del todo necesario desarrollar nuevas investigaciones que, partiendo del trabajo con corpus documentales amplios y fiables, permitan ir conociendo poco a poco las características que presenta la lengua española utilizada en la Guatemala colonial, de modo que sea posible describir con cierto detalle el desarrollo que ha experimentado durante este periodo histórico.

2. Como forma de contribuir al objetivo que se acaba de mencionar, el presente estudio tiene como propósito fundamental aportar algunos datos acerca de la diacronía del español guatemalteco por medio del análisis de la situación que presenta el nivel fónico de esta variedad en el siglo XVIII; en concreto, las cuestiones que se pretenden responder en estas páginas son dos: por un lado, si los fenómenos fónicos presentes en la documentación dieciochesca permiten sostener la hipótesis de que se ha producido ya en estos momentos la dialectalización de este nivel lingüístico, entendida como el 'resultado de los procesos de selección normativa que conllevan la imposición (o el rechazo) de determinados fenómenos que identifican y caracterizan geográficamente el español de una región' (Ramírez Luengo, en prensa 1); por otro -y en clara relación con lo anterior- determinar el grado de semejanza que existe desde el punto de vista fonético-fonológico entre el español del Setecientos y el que diversos estudiosos (Alvar 1980; Herrera Peña 1993; Lipski 1996: 280-285; Utgård 2010) describen para la Guatemala actual.

En cuanto al corpus que se ha utilizado para el análisis, se trata de una antología documental (Ramírez Luengo 2017) compuesta por 79 textos redactados entre 1690 y 1810 en diversas localidades de las tierras altas guatemaltecas, tales como Chiquimulilla, San Miguel Petapa o Santa Catarina Mita, pero muy especialmente las dos capitales coloniales, Santiago de los Caballeros y Nueva Guatemala de la Asunción ${ }^{1}$. Por lo que respecta a su tipología textual, se ha intentado que los materiales se aproximen en la medida en lo posible al polo de la inmediatez comunicativa (Oesterreicher 2004: 752-753), y eso ha determinado la inclusión predominante de denuncias e interrogatorios inquisitoriales, así como en menor medida escritos de otra naturaleza, como por ejemplo un conjunto de cartas privadas de mayor o menor formalidad; en todo caso,

1 El clarísimo predominio de las capitales en el corpus responde a una serie de razones históricas que se plantean en Ramírez Luengo (2017) y que determinan que "la implantación de la lengua española en Guatemala fue menos efectiva que en cualquier otra zona de Centroamérica, y se limitó, durante la época colonial, a un puñado de núcleos urbanos" (Lipski 1996: 280). 
cabe decir que se trata de escritos que, por sus propias características, favorecen la aparición de fenómenos de los diversos niveles de la lengua que resultan dialectalmente más marcados, y de ahí su evidente interés a la hora de reconstruir la variedad de español que se emplea en Guatemala a lo largo del siglo XVIII².

Por otro lado, es importante señalar también que la metodología que se emplea en este análisis parte de la constatación de que los fenómenos fónicos dejan su huella en los textos en forma de diversas cacografías que rompen las tendencias gráficas propias de la época (Frago 2002: 20-37)33; se procurará, por tanto, detectar en primer lugar todos los rasgos escriturarios que se salen de tales tendencias para aportar posteriormente una interpretación fónica que los explique y justifique, de modo que su análisis sistemático posibilite describir con un alto grado de certeza las principales características que presenta este nivel lingüístico en las coordenadas diatópicas y cronológicas que enmarcan el estudio.

3. Teniendo en cuenta, pues, lo descrito más arriba, es posible señalar en primer lugar la aparición en el corpus de una serie de grafías que reflejan la existencia, también en esta época, de ciertos fenómenos habituales hoy en la oralidad, tales como las simplificaciones de vocales de timbre similar por contacto, bien en interior de palabra (lerá; doc. 23; ler, doc. 35; cren, doc. 63) o bien entre vocablos distintos -entre otros, bolbiaser 'volvía a hacer', destar 'de estar' (doc. 15), del 'de él' (docs. 38, 72), nobstante (doc. 63), quieresaminar (doc. 76) y los frecuentísimos casos de la amalgama deste (docs. $4,8,11,16,17,24,25,35,39,72$, etc.) ${ }^{4}$-, la asimilación de la nasal implosiva a la consonante siguiente (comfesor, doc. 5; em presencia, doc. 14; emferma, doc. 26, comfesonario, ymformarme, doc. 63; emmendar, docs. 21, 35, 61; immediato, docs. 33, 49, 71; immaculada, docs. $47,51^{5}$ ) o la modificación de la consonante final de ciertos xenismos (Abrahan, doc. 51); en todos los casos se trata, por supuesto, de fenómenos comunes en el español, de manera que, si bien su interés dialectal es escaso, resulta con todo importante señalar su presencia en el corpus por reflejar, en palabras de Frago (1999: 209-210), "la connivencia entre lo fónico

2 Para mayores precisiones sobre el corpus utilizado en el estudio, véase la descripción del mismo que se recoge en Ramírez Luengo (2017).

3 Como en otros trabajos (a manera de ejemplo, Ramírez Luengo, en prensa 2), se entienden en esta ocasión por tendencias gráficas los "conjuntos de soluciones gráficas aceptadas socialmente en un momento concreto" (Ramírez Luengo 2012a: 168).

4 A este respecto, se registra también en Guatemala la evolución escrituraria que se señalaba previamente para el caso salvadoreño (Ramírez Luengo, en prensa 2), de manera que, mientras que en los dos primeros cortes cronológicos (1690-1730 y 1731-1770) el reparto entre las dos opciones se mantiene casi al 50\% -66 casos amalgamados frente a 67 separados y 11 amalgamados y 12 no amalgamados respectivamente-, en la última parte del corpus se descubre una clara decantación por la opción moderna, que se recoge en 23 ocasiones (95.83\%) frente a un único ejemplo amalgamado (4.16\%). Es necesario concluir, por tanto, que "al fenómeno fónico se superponen cuestiones relacionadas con las propias preferencias gráficas de cada momento” (Ramírez Luengo, en prensa 2), cuestión que es necesario tener en cuenta para la mejor interpretación de estas grafías en los textos del siglo XVIII.

5 Cabe indicar que, frente a la obvia explicación fonética de los casos de labiodental, estas últimas asimilaciones del grupo [nm] tienen una interpretación más compleja, habida cuenta de la prescripción de la grafía $<$ mm $>$ en tal contexto por parte de algunos ortógrafos de la Centuria Ilustrada; en todo caso, es importante mencionar que los usos 'asimilados' conviven a lo largo de todo el periodo con otros que no muestran tal fenómeno (enmendar, docs. 9, 50, 71; inmediato, doc. 43,44,56), e incluso con ejemplos aislados de simplificaciones gráficas (emendar, doc. 23, doc. 79) que se pueden interpretar como evidentes ejemplos de la simplificación, propia de la oralidad, de ambas consonantes en un contexto tan favorecedor como este. 
y lo grafémico"6 $y$, por tanto, por confirmar la utilidad, para los objetivos de este estudio, de una metodología como la propuesta en estas páginas.

3.1. Por lo que se refiere al vocalismo, quizá una de las cuestiones más sobresalientes teniendo en cuenta la época del corpus sea la práctica inexistencia de casos de vacilaciones en las vocales átonas: en efecto, los muy escasos ejemplos registrados -en concreto, cinco $^{7}-$ se descubren en su práctica totalidad durante la primera parte del periodo analizado (cerujano, sosodicha, doc. 19; aduleció, doc. 33) y en ningún caso van más allá de 1752 (irección, doc. 44), con la única excepción de un ympricionadas 'impresionadas' de 1791 (doc. 63) que se descubre en una carta redactada por una mujer caracterizada como mano inhábil. De este modo, parece posible concluir que este fenómeno, muy limitado ya desde las postrimerías del siglo XVII, termina por desaparecer de la pronunciación -o al menos de la escritura- culta del español guatemalteco en la primera mitad del Setecientos, mientras que, en el caso de otros niveles socioeducacionales, la alternancia se mantiene durante un periodo ligeramente mayor ${ }^{8}$.

Junto a estas escasas muestras de vacilaciones vocálicas, se registran también otras que en apariencia son semejantes, pero que en realidad deben ser explicadas a partir de factores diferentes como son, por ejemplo, la conservación, aún en el siglo XVIII, de las variantes medievales y áureas de determinados verbos: tal parece ser el caso de los ejemplos de escribir y recibir con /e/ átona (Frago 1999: 46, 114) que se descubren -en convivencia con la variante modernahasta mediados de la centuria (escrevir, docs. 23, 29, 37; recebido, doc. 11, recebi, doc. 29, recevimos, doc. 41), momento en el que desaparecen a favor de las formas con /i/, o las apariciones de inviar (imbio, doc. 1; imbiarle, doc. 26), que Frago (2002: 493) considera muy frecuente durante el quinientos y que en el español de Guatemala se registran hasta 1730, en una situación muy semejante a la que se ha descrito para estos momentos en numerosas zonas del Nuevo Mundo, y entre ellas también algunas centroamericanas como Honduras o El Salvador (Ramírez Luengo 2004-5: 59, en prensa 2) $)^{9}$.

6 Por si hubiera alguna duda sobre tal connivencia y el origen puramente fónico de estos usos gráficos anómalos, conviene recordar que muchos de los ejemplos anteriores aparece precisamente en documentos generados por autores que pueden ser definidos como manos inhábiles -a manera de ejemplo, docs. 15, 63, 76-, esto es, por hablantes que, por tener una menor destreza escrituraria y un más escaso dominio de las convenciones sociales que rigen esta práctica, dejan traslucir mejor en sus textos fenómenos propios de la oralidad (Petrucci 2000: 69).

7 A estos casos se suman otros cuya interpretación es más dudosa: se trata, en concreto, de dos apariciones del apellido Jaúregui con /i/ (Sabaljáurigui, doc. 62; Jáurigui, doc. 64) -que quizá se deban entender no tanto como vacilaciones, sino más bien como muestra lexicalizada de las modificaciones que en ocasiones sufre la onomástica peninsular en su traslado a América- y dos ejemplos de difinitorio (docs. 18, 68), voz que el DLE (2017: s.v. definitorio) recoge con /e/ pero cuya aparición con /i/ resulta frecuente en el Nuevo Mundo durante los siglos XVII y XVIII, a juzgar por los datos de CORDIAM y CORDE (en concreto, 6 casos con /e/ frente a 15 con /i/).

8 En este sentido, la confirmación de que en la Guatemala del Setecientos existe variación sociolingüística en este punto del sistema permite entender mejor las diferencias registradas al respecto entre el memorial de un sacerdote criollo -caracterizado por la ausencia total de este fenómeno (Pérez Cordón y Ramírez Luengo 2007: 100-101) - y diversos documentos notariales, en los que, por el contrario, aparece de forma relativamente frecuente (Ramírez Luengo 2006: 263).

9 Cabe mencionar que el proceso de cambio vocálico que se acaba de describir parte en todas las ocasiones de una situación de convivencia de variantes y una decantación progresiva hacia la forma moderna, tal y como demuestran los porcentajes de esta que se ofrecen para los tres cortes cronológicos en que se divide el corpus (1690-1730, 1731-1770, 1771-1810): recibir: $34.78 \%>33.33 \%>100 \%$; escribir: $73.68 \%>80 \%>100 \%$; enviar: $62.5 \%>100 \%>100 \%$. 
Por otro lado, si la consolidación del vocalismo átono es prácticamente total desde los mismos inicios del periodo analizado en estas páginas, no sorprende que esta sea también la situación que se registra en el caso de las vocales tónicas: en efecto, más allá de un ejemplo de vacilación /i/>/e/ que quizá no pase de mero lapsus cálami (legétima, doc. 12), lo único destacable al respecto es el mantenimiento de variación en un vocablo específico, mismo, o más bien, la convivencia de las variantes etimológica mesmo y moderna mismo durante las primeras décadas del siglo y el posterior triunfo, a partir de 1750, de la forma con /i/, en un cambio que, una vez más, no solo es general en el español, sino que además parece coincidir en su cronología con lo que se ha indicado ya para otras zonas de América (Sánchez Méndez 1997: 61; Donni de Mirande 2004: 63) $)^{10}$.

En cuanto a los diptongos, de nuevo se debe señalar la existencia en el corpus de una situación muy cercana a la actual, habida cuenta de que solo se descubren en él una monoptongación en un contexto altamente favorecedor como son los compuestos de veinte (ventidos, doc. 10) y algunos casos esporádicos de diptongación irregular, muy especialmente -aunque no solo- en el caso del verbo entregar (ausiensia; doc. 7; entriegue, doc. 40; entrieguen, doc. 41; entriego, doc. 51); se trata, por tanto, de muestras guatemaltecas de fenómenos muy habituales en numerosas áreas americanas (y centroamericanas) del Siglo Ilustrado (Frago 1999: 210; Quesada Pacheco 2009: 86; Ramírez Luengo, en prensa 2) que evidencian una vez más un comportamiento común y compartido en el español del continente al que la variedad de esta zona no es en modo alguno ajena.

Frente a estos aspectos más o menos generales en la época, es interesante señalar la presencia de rasgos diatópicamente más restringidos que caracterizan hoy al español de Guatemala: a manera de ejemplo, el corpus arroja algunas cacografías de elisión vocálica (en grasi de Dios, la caus de, todopoderos por; doc. 15; don Jos María, doc. 75) que probablemente se puedan considerar muestras dieciochescas de la tendencia al relajamiento o elisión de vocales en posición átona que se registra esporádicamente en la región (Alvar 1980: 187; Utgård 2010: 53) ${ }^{11}$, así como muestras del refuerzo antihiático -varios casos de leiya en el documento 79- que tampoco es desconocido en la zona (Lipski 1996: 283; Quesada Pacheco 1996: 103) y cuya presencia, a la luz de estos casos, se puede adelantar al menos hasta comienzos del siglo XIX. Por otro lado, resulta relevante señalar también la inexistencia prácticamente total de ejemplos de diptongación de los hiatos -el único caso lo constituye aunde 'adonde' (doc. 39), habida cuenta de que el resto son cuanto menos dudosos ${ }^{12}$-, algo sin duda sorprendente no solo por tratarse de un fenómeno

10 En realidad, los datos demuestran que, ya desde finales del siglo XVII, la forma mismo es claramente mayoritaria, al aparecer en el 71.66\% de las ocasiones (43 casos frente a 17); con todo, mesmo se mantiene de forma ocasional durante el periodo 1731-1770 -tres apariciones y un 12.5\% del total-, para desaparecer en 1753 (doc. 46), de manera que en el último corte cronológico solo se descubre la moderna mismo, en un total de 55 ocasiones.

11 Cabe indicar, además, que la mayoría de estas pérdidas vocálicas se produce junto a /s/, como es sabido un contexto favorecedor del fenómeno; tampoco carece de relevancia señalar su presencia en textos de manos inhábiles (docs. $15,75,76)$. Por otro lado, se descubre también la aparición -una vez más, en un texto femenino de una mano inhábilde un caso de adición vocálica que sirve para romper un grupo culto consonántico (capetiverio, doc. 63), lo que muestra de nuevo la ya señalada connivencia entre lo fónico y lo gráfico y ratifica, una vez más, la pertinencia de la metodología aplicada en el estudio.

12 Como ambiguo puede calificarse el caso de crio (doc. 51), dado que la inexistencia en la época de una clara diferenciación entre los verbos crear/criar y el contexto en que se registra el ejemplo ( $O$ virgen Maria por aquel que te Crio, i nacio de vos venza yo a mis amigos, $i$ enemigos) impide saber cuál de los dos se está empleando. Por otro lado, las 
muy general en el español americano del momento (Ramírez Luengo 2007: 41), sino también por su presencia en la variedad guatemalteca actual (Alvar 1980: 187), lo que obliga a plantear la posibilidad de que, en el caso concreto de Guatemala, tal fenómeno no se dé en la época colonial y, por lo tanto, se desarrolle ya durante el siglo XIX ${ }^{13}$.

3.2. De este modo, el análisis del vocalismo de los documentos del corpus permite constatar que el español guatemalteco de la época se caracteriza por presentar, junto a fenómenos propios del siglo XVIII, otros más restringidos desde el punto de vista diatópico que sirven para identificar esta variedad en el Setecientos y que se mantienen hasta la actualidad; pues bien, se puede adelantar ya que la descripción del consonantismo ofrece unas conclusiones muy similares a estas, aunque indudablemente de mayor trascendencia a la hora de establecer el perfil dialectal de esta variedad geográfica, tal y como se podrá observar en los siguientes párrafos.

En primer lugar, es necesario mencionar la aparición prácticamente generalizada en el corpus de confusiones gráficas que demuestran la inexistencia, ya en estos momentos, de oposición fonológica entre las sibilantes $/ \mathrm{s} /$ y $/ \theta /{ }^{14}$ : en efecto, con la excepción de un escaso número de documentos debidos a cinco autores -quizá peninsulares emigrados ${ }^{15}$-, la inmensa mayoría de los textos estudiados presenta abundantes cacografías que manifiestan que la indistinción de sibilantes, si no absoluta, al menos es un fenómeno de muy notable extensión en el español de la zona (meztiza, doc. 1; pretenciones, doc. 5; aprendis, doc. 13; entreserrado, doc. 28; jusgado, doc. 41; casique, doc. 59; perzona, doc. 78); por supuesto, tal extensión del fenómeno en la Guatemala del siglo XVIII no resulta inesperada por cuanto coincide con lo que se sabe acerca de su rápida generalización por toda la América hispana (Ramírez Luengo 2007: 33-34), pero en todo caso es importante señalar

grafías de Joan y Joana (docs. 1, 13, 14) constituyen un uso gráfico habitual en estos momentos para los antropónimos 'Juan' y 'Juana', de manera que en modo alguno se pueden considerar ultracorrecciones relacionadas con la diptongación de los hiatos; lo mismo sucede en el caso de Goatemala/Guatemala, habida cuenta de que la primera forma es el nombre primitivo de la ciudad y el reino (Ramírez Luengo 2006: 263) y que solo a lo largo del siglo XVIII cede ante la forma moderna con $/ \mathrm{u} /$, tal y como se evidencia en los porcentajes de uso de esta que se extraen del corpus: $48.48 \%$ $(1690-1730)>72.72 \%(1731-1770)>96.42 \%(1771-1810)$.

13 Esta hipótesis se ve reforzada por el hecho de que ni siquiera los escritos de las manos inhábiles -en los que afloran de forma muy frecuente numerosos rasgos de la oralidad de la época- muestran ejemplos evidentes de tal diptongación, lo que parece avalar la idea de su inexistencia en el español guatemalteco del momento.

14 Al igual que se señala en Ramírez Luengo (en prensa 2), es preferible hablar de inexistencia de distinción y no tanto de seseo, habida cuenta de que actualmente es posible registrar en el país tanto casos de este fenómeno como de ceceo (Alvar 1980: 198, Utgård 2010: 61), de manera que es imposible determinar la realización fonética concreta -esto es, siseante o ciceante- de cacografías como comenso (doc. 10), imprecion (doc. 47) o payzanos (doc. 69). Es muy probable, con todo, que ambos usos convivieran ya en el siglo XVIII, al igual que conviven actualmente, y a este respecto son especialmente interesantes los documentos 4, 8, 9, redactados por Miguel de Carranza y Córdoba, porque en ellos las grafias confundidoras se reducen al antropónimo Luiza (doc. 4) y al apodo Zoletera (docs. 8, 9); teniendo en cuenta que la ausencia de confusiones generalizadas parece apuntar a que este escribano es distinguidor, el hecho de que sus únicos deslices gráficos aparezcan en nombres propios y apodos que probablemente escucha al dictado -y, por tanto, reproduce como le son pronunciados- quizás esté confirmando la existencia de usos ceceantes en estos momentos, si bien la absoluta certeza al respecto resulta imposible por medio de la metodología que se está utilizando.

15 Tal es la situación de Juan Infante (doc. 68), que es identificado en otro de los textos como castellano (doc. 69); más interesante, sin embargo, es el caso de José Mariano Jáuregui (doc. 64), guatemalteco de nacimiento (Dym 2000: 142) que muestra un perfecto uso de las grafías mencionadas, lo que obliga a plantearse si tal ausencia de cacografías no es quizá muestra de una pronunciación distinguidora que se mantiene aún en el siglo XVIII en determinados grupos sociales de Guatemala, tal y como sugiere Guitarte (1991) en el caso de otras zonas americanas del Ochocientos. 
su aparición en el corpus no solo por ser uno de los rasgos sobresalientes en la caracterización fónica del español del Nuevo Mundo, sino también porque corrobora las conclusiones a las que se había llegado al respecto en trabajos anteriores sobre la historia del español guatemalteco (Pérez Cordón y Ramírez Luengo 2007: 100; Ramírez Luengo 2006: 265).

Frente a lo anterior, muy otra parece ser la situación del yeísmo en el corpus, pues si bien hoy constituye un rasgo generalizado en el país (Alvar 1980: 194; Herrera Peña 1993: 4; Utgård 2010: 76), su escasa atestiguación en estos documentos permite postular el carácter incipiente del fenómeno en la época, así como su progresiva expansión a lo largo del siglo XVIII: en concreto, se descubre en un único documento del segmento temporal 1690-1730 (hayo 'halló; doc. 7), en uno del segundo (1731-1770) (yamada, ayado, doc. 33) y cinco de la última parte de la centuria (1771-1810) (lellese, doc. 61; valla 'vaya', doc. 63; apollan, doc. 69; mallor, doc. 76; yegada, doc. 79$)^{16}$, lo que parece evidenciar un claro proceso de expansión, al pasar del 3.12\% de la primera etapa al $17.85 \%$ de la última. De este modo -y a pesar de que la no aparición de grafías yeístas no implica necesariamente la inexistencia de este fenómeno en los escriptores-, los datos expuestos hasta ahora permiten sostener, por un lado, que el yeísmo está aún lejos de generalizarse en la Guatemala dieciochesca; por otro, que se trata de una pronunciación presente ya en la zona y que muy probablemente cuenta con una distribución de carácter sociolingüístico difícil de precisar, lo que no solo corrobora los resultados de estudios anteriores sobre este asunto (Herrera Peña 1993: 3; Pérez Cordón y Ramírez Luengo 2007: 100; Ramírez Luengo 2006: 265-6), sino que además aporta precisiones cronológicas acerca de su proceso de generalización en la variedad de español propia del país.

Junto a estos dos, el tercer fenómeno más caracterizador del español guatemalteco es sin duda la retención de la pronunciación sibilante de la /-s/ implosiva, que en general no sufre la elisión propia de otras zonas de Centroamérica (Alvar 1980: 199; Lipski 1996: 284; Quesada Pacheco 1996: 104; Utgård 2010): de este modo, no sorprende que en el corpus prácticamente no aparezcan cacografías que atestigüen la aspiración de tal fonema y que los escasos usos ánomalos se puedan explicar como resultado de meros lapsus cálami ${ }^{17}$, así como que la única excepción a lo anterior sea un prebiteros (doc. 35) documentado en 1735 en el departamento de Jutiapa, esto es, en la zona (sur)oriental del país donde Herrera Peña (1993: 3) registra actualmente el debilitamiento o lenición de dicho fonema; es posible concluir, por tanto, que los datos del corpus ofrecen en este punto una situación muy semejante a la que existe hoy en día en la región, de manera que resulta muy probable que, por lo que se refiere a este fenómeno, la situación actual se pueda retrotraer al menos hasta el mismo siglo XVIII.

Por su parte, en relación con la aspiración de /s/ se encuentran también los relajamientos y caídas de fonemas que configuran un consonantismo débil: aunque es verdad que en general

16 Cabe indicar que este aumento de documentos con cacografías yeístas se acompaña también, naturalmente, de un aumento de los escriptores que traslucen tal fenómeno fonológico, que pasa del 5.88\% de la primera etapa (uno de 17, en concreto N. Roxel) al $8.33 \%$ de la segunda (uno de doce, J. de Pineda) y al $26.31 \%$ de la última fase (cinco de 19 , a saber: J. de Zepeda, P. A. Gordon, A. Alonso Cortés, A. Matute y A. González).

17 Tal es el caso de costeste 'conteste' (doc. 22) y existo 'excitó' (doc. 47), cuyas <s> ultracorrectas pueden guardar relación con la presencia de otra $<\mathrm{s}>\mathrm{y}$ de $<\mathrm{x}>$ en los términos en que aparecen; por otro lado, es probable que el caso de tapandole la boca (doc. 14) -donde le cuenta con un referente plural- responda a motivaciones morfológicas y se deba entender como un ejemplo de la pérdida del morfema de plural en el pronombre de dativo, algo relativamente frecuente en el español desde antiguo (Gómez Seibane 2012: 71-2). 
el corpus se caracteriza por la escasez de tales fenómenos, lo cierto es que es posible registrar de manera esporádica la desaparición de las oclusivas sonoras/b/y de forma más frecuente /d/, tanto en posición final (verda, doc. 53) como muy especialmente intervocálica (ahundantemente 'abundantemente', doc. 38; cantides 'cantidades', doc. 39; aunde/aonde 'adonde', docs. 39, 76) ${ }^{18}$; cabe indicar que este fenómeno -que se acompaña también de un caso aislado de elisión de /r/ intervocálica (declase 'declarase'; doc. 39) ${ }^{19}$ - se descubre preferentemente, aunque no de manera exclusiva, en los documentos de las manos inhábiles, lo que quizás demuestre su especial incidencia en el habla de los grupos más populares, si bien el corpus no permite llegar a conclusiones sólidas al respecto ${ }^{20}$. Esta hipótesis es también aplicable para los casos de elisión de la /n/ implosiva, que se concentran en los textos redactados por una mujer englobable dentro de las manos inhábiles (denusio, doc. 75; co mi 'con mi', estaco 'estanco, ynportate, doc. 76) y que muy probablemente reflejen la velarización y elisión de este fonema en tal posición ${ }^{21}$, proceso que está ampliamente documentado en el español actual de la zona (Alvar 1980: 263; Herrera Peña 1993: 4; Lipski 1996: 284; Utgård 2010: 68).

Por otro lado, es posible señalar también la presencia en el corpus de algunas grafías que se descubren de forma muy ocasional, pero que no carecen de interés por cuanto manifiestan la vigencia en la época de algunos fenómenos de interés: por un lado, un caso aislado de guerfanos (doc. 15) demuestra el empleo guatemalteco del refuerzo velar del diptongo [we], que aparece frecuentemente en la época en diferentes áreas del mundo hispánico (Ramírez Luengo 2013: 57-8, en prensa 2) y cuya generalidad le resta interés dialectal, si bien contribuye a describir de forma más precisa las características fónicas de esta variedad lingüística ${ }^{22}$; por otro, se descubren otros usos que parecen avalar la existencia dieciochesca de fenómenos muy identificadores del español guatemalteco actual (Alvar 1980: 204-7; Lipski 1996: 283-4; Utgård 2010: 57, 69-70), tales como la realización no aproximante de las oclusivas sonoras que quizá represente

18 También se documenta en el corpus la desaparición de la /g/ intervocálica en el caso del vocablo agora, que muestra la convivencia de las formas antigua y moderna en el periodo 1690-1730 -si bien con una clara preferencia ya por esta última: $92.30 \%$ de las ocasiones, 24 de 26 - y un dominio absoluto de ahora a partir de ese momento, algo que resulta privativo de Guatemala, sino que se produce también en otras zonas del dominio hispánico de la época (Ramírez Luengo 2013: 65-6); con todo, el carácter propiamente léxico de este proceso de cambio invita a considerarlo de forma autónoma y no equipararlo, por tanto, a las muestras de consonantismo débil que se han señalado más arriba.

19 En realidad, más allá de la alternancia léxica entre proprio (docs. 28, 52, 62) y propio (doc. 6), el fenómeno más frecuente en el caso de las vibrantes son las confusiones en el uso de $<\mathrm{r} / \mathrm{rr}>$ intervocálicas, según se ve en ejemplos como, entre otros, hariero, sero (doc. 23), guera (doc. 63), Vidaure (doc. 75) o bario (doc. 76); aunque cabe la posibilidad de que se traten de meros usos gráficos sin trascedencia fónica (Frago 2007: 155-156), no es del todo descartable que sean reflejo del rico polimorfismo que presenta este fonema actualmente, con variantes alveolares y asibiladas (Utgård 2010: 69-76), lo que demostraría la vigencia de una situación semejante a la actual ya en la Centuria Ilustrada.

20 De hecho, esta parece ser la situación actual en el país, según se desprende de algunos comentarios presentes en el trabajo de Alvar (1980: 193).

21 En el caso de Costa Rica Quesada Pacheco (2009: 145-146) interpreta de esta manera elisiones gráficas semejantes a estas que se registran en documentos coloniales, de manera que no es difícil pensar que, en el caso guatemalteco, estas cacografías respondan al mismo proceso.

22 Algo semejante ocurre con el mantenimiento de la /f-/ inicial en fecho (docs. 6, 7, 37, 54), que se registra de forma habitual en determinadas fórmulas notariales rutinizadas y cuya presencia se debe interpretar como un rasgo propio de la tipología textual -en concreto, de la documentación notarial- a la que pertenecen los escritos donde se emplea; por supuesto, en ningún caso es un fenómeno propio de Guatemala, pues aparece en múltiples zonas del dominio hispánico de este mismo periodo, también algunas centroamericanas como El Salvador (Ramírez Luengo, en prensa 2). 
la grafía puplicamente (doc. 39) ${ }^{23}$, el empleo del alófono laríngeo o débil [h] del fonema /x/ (contrahese, doc. 23; guegos 'juegos', doc. 39; mongas 'monjas', doc. 53) o -de forma mucho más discutible- la existencia de la [̌r] asibilada que quizá explique la pérdida vocálica que se descubre en trtar (doc. 63) $)^{24}$.

Finalmente, no se puede terminar un análisis de la configuración fónica sin describir la situación en la que se encuentran los denominados grupos cultos, esto es, las secuencias consonánticas que cuentan (de forma general) con una oclusiva en posición implosiva: como es de sobra conocido, tradicionalmente se ha considerado que la recuperación de tales grupos se produce a partir del siglo XVIII como resultado de la preceptiva que al respecto propone la Real Academia Española (Lapesa 1985: 421); sin embargo, diversos estudios sobre áreas españolas y americanas (Ramírez Luengo 2010, 2013: 61, 2004-5: 58, 2012b: 297, en prensa 2) demuestran que, en realidad, el proceso de recuperación comienza en el siglo XVII y que, por tanto, para la Centuria Ilustrada está ya ampliamente difundido y resulta claramente mayoritario ${ }^{25}$, algo que ya se había señalado en el caso del español guatemalteco (Pérez Cordón y Ramírez Luengo 2007: 101; Ramírez Luengo 2006: 267-8) y que se ve ampliamente confirmado ahora por los datos que se extraen de este corpus (tabla 1):

\begin{tabular}{|l|c|c|c|}
\hline GRUPO $^{*}$ & $1690-1730$ & $1731-1770$ & $1771-1810$ \\
\hline Biconsonánticos & $147 / 2(98.65 \%)$ & $136 / 3(97.84 \%)$ & $165 / 3(98.21 \%)$ \\
\hline Triconsonánticos & $36 / 0(100 \%)$ & $43 / 6(87.75 \%)$ & $52 / 7(88.13 \%)$ \\
\hline TOTAL & $183 / 2(98.91 \%)$ & $179 / 9(95.21 \%)$ & $217 / 10(95.59 \%)$ \\
\hline
\end{tabular}

* Por lo que se refiere a los grupos biconsonánticos, se incluyen en este apartado los siguientes: [p+cons.], [k+cons.], [b+cons.], [d+cons.], [g+cons.] y [m+cons.]; en el caso de los triconsonánticos, se trata de [ks+cons.], [ns+cons.] y [bs+cons.]. Cabe indicar que la metodología que se ha seguido en este análisis es la propuesta en Ramírez Luengo (2010), de manera que se remite a este estudio para mayores especificaciones al respecto.

Tabla 1: Distribución de la variantes plena/simplif. en los grupos cultos (1690-1810)

23 Téngase en cuenta, de todas formas, que el ejemplo resulta cuanto menos problemático, pues en posición intervocálica -como es el caso del corpus- la pronunciación fricativa suele ser la habitual (Quesada Pacheco, 1996: 103); cabe mencionar que un ejemplo semejante (casticado) se descubre en el español salvadoreño de esta misma época (Ramírez Luengo, en prensa 2).

24 A este indicio de la existencia ya en esta época de la [̌r] asibilada se deben sumar las confusiones gráficas de $<$ r/ rr> intervocálica que se han mencionado anteriormente en la nota 19, todo lo cual quizá permita postular la presencia de este alófono de la vibrante ya en el Siglo de las Luces, al menos como hipótesis que será necesario confirmar con análisis posteriores.

25 Cabe recordar que este proceso está determinado léxicamente (Ramírez Luengo 2010: 254), de manera que, junto a la más generalizada recuperación consonántica, se producen también procesos de simplificación en términos concretos: a manera de ejemplo, esto es lo que se descubre en el corpus con escrito, término para el cual conviven la forma plena (docs. 3, 23, 49) y simplificada (docs. 2, 21, 37) a lo largo del siglo, pero con una clara progresión hacia la imposición de esta segunda, que pasa del 75.60\% del primer periodo (31/41) al $90.90 \%$ del segundo (10/11) y al 100\% del último (21/21); algo semejante parece producirse también con substancia/sustancia (docs. 2, 9, 21; docs. 31, 50), si bien la escasez de ejemplos impide esbozar claramente su evolución. 
Así pues, los porcentajes que se acaban de presentar permiten extraer varias conclusiones de relevancia que explican mejor la historia de estos elementos y de su generalización en el español guatemalteco: por un lado, salta a la vista que el empleo de la variante plena en esta variedad diatópica no es una innovación del siglo XVIII, sino que se produce en momentos anteriores, habida cuenta de los altos porcentajes de uso, de más del 95\%, que se registran en todos los cortes cronológicos analizados; por otro, es importante señalar también que el proceso de recuperación en sí está determinado por la naturaleza del grupo consonántico que se considere, y que parece estar ligeramente más avanzado en el caso de los grupos biconsonánticos ${ }^{26}$; por último, si se tiene en cuenta que la mayor parte de las simplificaciones aparecen en los escritos de las manos inhábiles (satisfacion, doc. 15; espiro, doc. 39; esaminar, doc. 76), es necesario concluir que, al igual que sucede hoy en día, también en el Setecientos este fenómeno está parcialmente determinado por factores de tipo sociolingüístico, aspecto que será necesario tener en cuenta para futuros análisis que se ocupen de esta cuestión. De este modo, los datos demuestran que la variedad guatemalteca presenta en este punto concreto del sistema una situación virtualmente idéntica a la que se descubre en otras zonas del dominio hispánico en la época y, por tanto, que comparte -desde el punto de vista de los procesos, pero también de las cronologías- las tendencias generales que parecen caracterizar la evolución fónica del español durante la Centuria Ilustrada.

4. Así pues, el análisis de las grafías presentes en el corpus base de este estudio permite establecer ya una serie de conclusiones de interés para el mejor conocimiento de la historia del español guatemalteco, y más concretamente de la configuración fónica que presenta durante la última parte del periodo colonial.

A este respecto, los datos permiten en primer lugar señalar la presencia en estos momentos de una serie de características que identifican al español del siglo XVIII y que, si bien poseen escaso interés desde el punto de vista dialectal, sirven para dar una imagen más completa de la situación que presenta esta variedad en la época: en este sentido, son especialmente destacables las muy escasas vacilaciones que se registran en el vocalismo átono, los procesos de cambio vocálico que afectan a ciertos elementos (mesmo, escrebir, recebir, inviar), las diptongaciones y monoptongaciones anómalas, la presencia del refuerzo velar ante [we] o la clarísima preferencia por la reposición de las oclusivas en los denominados grupos cultos, todo lo cual demuestra -como, por otro lado, era de esperar- la participación de la variedad guatemalteca del español en los grandes procesos de evolución que caracterizan a la lengua en la sincronía estudiada.

Junto a lo anterior, el análisis evidencia también la presencia en el corpus de ciertos fenómenos fónicos que identifican actualmente al español de Guatemala desde el punto de vista diatópico y cuya presencia en esta variedad, a la luz de estos datos, se puede retrotraer al menos hasta el Setecientos; en este caso, son especialmente destacables el relajamiento de vocales

26 Un análisis más preciso demuestra que, en realidad, ni siquiera todos los grupos que pertenecen a cada una de las categorías se comportan de la misma manera: por lo que se refiere a los biconsonánticos, por ejemplo, solo [k + cons.] presenta casos de simplificación, mientras que los otros (es decir, [b+cons.], [p+cons.], [d+cons.], [g+cons.] y [m+cons.]) mantienen de manera constante el uso pleno en todos los periodos analizados; por lo que se refiere a los triconsonánticos, solo [ks+cons.] ofrece algunos ejemplos de simplificación, de manera que no sorprende que sea precisamente esta secuencias la que ofrece la práctica totalidad de casos de ultracorrección gráfica (excasamente, excarnio, doc. 28; exfuerso, doc. 38; contextar, doc. 40; expontaneamente, doc. 53; expontanea, doc. 74). 
átonas, el refuerzo antihiático, la confusión de sibilantes, el consonantismo fuerte -esto es, sin caídas consonánticas y con /-s/ implosiva sibilante- que en general se descubre en los textos y la -discutible- realización no aproximante de las oclusivas sonoras, así como la imposición de determinados alófonos como la /-n/ implosiva velar, la aspirada [h] del fonema velar /x/ o, con más dudas, la [̌r $[$ asibilada. Al mismo tiempo, es importante mencionar también otras cuestiones que se desprenden de este estudio como son, por ejemplo, la aparente inexistencia de diptongación de los hiatos y el estado incipiente en que se encuentra el yeísmo, las cuales cobran especial relevancia histórica por cuanto demuestran que, por lo que se refiere al nivel fónico de esta variedad geográfica, no todo son coincidencias entre el siglo XVIII y la actualidad.

En definitiva, parece posible concluir estas páginas sosteniendo que para el periodo tardocolonial el español guatemalteco se caracteriza por contar ya con una configuración fónica que presenta un perfil dialectal muy marcado, en el que se incluyen, si no todos, al menos muchos de los fenómenos que actualmente se consideran más identificadores del país centroamericano; así las cosas, es muy probable que tal configuración, impuesta al menos desde las postrimerías del siglo XVII, dote a esta variedad diatópica de una fuerte personalidad en la Centuria Ilustrada, y que, en consecuencia, constituya -junto a cambios propios del Ochocientos que será necesario estudiar más adelante- la base de la innegable originalidad que, desde el punto de vista del fonetismo, presenta a día de hoy dentro de la dialectología del español de América.

\section{Referencias bibliográficas}

Alvar, M. (1980). Encuestas fonéticas en el suroccidente de Guatemala. In Norma lingüística sevillana y español de América (pp. 179-221). Madrid: Ediciones de Cultura Hispánica.

[CORDE]: Real Academia Española. Banco de datos CORDE: Corpus Diacrónico del español. En línea: $<$ http://www.rae.es> [Consulta: agosto, 2017].

[CORDIAM]: Academia Mexicana de la Lengua. Corpus diatópico y diacrónico del español de América. En línea: <http://www.cordiam.org> [Consulta: julio, 2017].

Donni de Mirande, N. E. (2004). Historia del español en Santa Fe del siglo XVI al siglo XIX. Buenos Aires: Academia Argentina de Letras.

Dym, J. (2000). A Sovereign State of Every Village: city, state and nation in independence-era Central America, ca. 1760-1850 (tesis de doctorado inédita). Nueva York: New York University.

Frago, J. A. (1999). Historia del español de América. Textos y contextos. Madrid: Gredos.

- (2002). Textos y normas. Comentarios lingüísticos. Madrid: Gredos.

. (2007). Tradición e innovación en el español americano de la Independencia. Romance Philology, 61(2), 147-191.

Gómez Seibane, S. (2012). Los pronombres átonos (le, la, lo) en el español. Madrid: Arco/Libros.

Guitarte, G. L. (1991). Seseo y distinción $s-z$ en América durante el siglo XIX. In Siete estudios sobre el español de América (pp. 107-125). México: Universidad Nacional Autónoma de México.

Hernández, E. (2008a). Aproximación al Vocabulario de la lengua cakchiquel o guatemalteca de fray Thomás de Coto (c. 1650) como fuente léxica del español. Bulletin Hispanique, 110 (1), 319-331. 
- (2008b). Indigenismos en el Vocabulario de la lengua cakchiquel atribuido a fray Domingo de Vico, ms. BNF R.7507. Revista de Filología Española, 88(1), 67-88.

Herrera Peña, G. (1993). Los idiomas hablados en Guatemala. Notas sobre el español hablado en Guatemala. Boletín de lingüistica y educación, VII (42), 1-4.

Lapesa, R. (1985). Historia de la lengua española. Madrid: Gredos.

Lipski, J. M. (1996). El español de América. Madrid: Cátedra.

Oesterreicher, W. (2004). Textos entre inmediatez y distancia comunicativas. El problema de lo hablado escrito en el Siglo de Oro. In R. Cano Aguilar (Coord.), Historia de la Lengua Española (pp. 729-769). Barcelona: Ariel.

Pérez Cordón, C.; \& Ramírez Luengo, J. L. (2007). El español de Guatemala en el siglo XVIII: un memorial de un criollo. In C. Pérez Cordón \& J. L. Ramírez Luengo (Eds.), El español en sus textos: Manual de comentarios lingüísticos y filológicos (pp. 99-108). Lugo: Axac.

Petrucci, A. (2000). Escrituras marginales y escribientes subalternos. Signo. Revista de historia de la cultura escrita, 7, 67-75.

Polo Cano, N. (2005). Algunos indigenismos léxicos en el español de Guatemala del siglo XVIII. In A. García Lenza \& A. Rodríguez Barreiro (Eds.), Res Diachronicae Virtual 4: El contacto de lenguas (pp. 185-202). En línea: <http://www.resdi.net> [consulta: agosto, 2017].

Quesada Pacheco, M. Á. (1996). El español de América Central. In M. Alvar (Dir.), Manual de Dialectología Hispánica, II. El español de América (pp. 101-115). Barcelona: Ariel.

- (2009). Historia de la lengua española en Costa Rica. San José: Universidad de Costa Rica.

Ramírez Luengo, J. L. (2004). Para una historia del español en Guatemala: notas sobre historia externa en el siglo XVIII. In M. Fuertes Gutiérrez, M. J. García Folgado \& J. L. Ramírez Luengo (Eds.), Res Diachronicae Digital 3: Estudios sobre el siglo XVIII (pp. 153-70). En línea:<http://www.resdi.net>.

- (2004-5). Contribución a la historia del español de Honduras: edición y estudio de documentos hondureños del siglo XVIII. Anuario de Letras, 42-43, 51-75.

. (2006). Aproximación al español de Guatemala en el siglo XVIII: algunas características fonético-fonológicas. Lexis, 30 (2), 259-272.

. (2007). Breve historia del español de América. Madrid: ArcoLibros

. (2010). La representación de los grupos cultos consonánticos en el español del Bilbao dieciochesco. In S. Gómez Seibane \& J. L. Ramírez Luengo (Eds.), Maestra en mucho. Estudios filológicos en homenaje a Carmen Isasi Martínez (pp. 241-256). Buenos Aires: Voces del Sur.

- (2012a). Notas sobre las tendencias gráficas del español colombiano en la época de las Independencias (1830). In J. L. Ramírez Luengo (Coord.), Por sendas ignoradas. Estudios sobre el español del siglo XIX (pp. 167-182). Lugo: Axac.

- (2012b). Una aportación a la historia de la lengua española en Nicaragua: algunos datos sobre el siglo XVIII. In Ma. T. García Godoy (Ed.), El español del siglo XVIII (pp. 293-312). Berna/Bruxelles/ New York: Peter Lang.

- (2013). Una descripción del español de mediados del siglo XVIII. Edición y estudio de las cartas de M. Martierena del Barranco (1757-1763). Lugo: Axac.

. (2017). Documentos lingüísticos de la Guatemala dieciochesca (1690-1810). Lugo: Axac.

. (en prensa 1). Aspectos metodológicos para el estudio histórico del léxico americano: conceptos, ejemplificación y tareas para el futuro. Moenia, 23, 2017. 
(en prensa 2). La configuración fónica del español salvadoreño en la época colonial (1650-1803). Boletín de la Real Academia Española.

Ramírez Luengo, J. L.; Aquino Melchor, S. A.; \& Ramírez Vázquez, J. J. (2017). Indigenismos léxicos en el español guatemalteco del siglo XVIII. Lengua y habla, 21, 111-125.

Sánchez Méndez, J. (1997). Aproximación histórica al español de Venezuela y Ecuador durante los siglos XVII y XVIII. Valencia: Tirant lo Blanch.

Taracena Arriola, A. (1985). El 'voseo' en la literatura guatemalteca en el siglo XIX. Anales de la Academia Guatemalteca de Geografía e Historia, 59, 29-35.

Utgård, K. (2010). El español de Guatemala. In M. A. Quesada Pacheco (Ed.), El español hablado en América Central. Nivel fonético (pp. 49-81). Madrid/Frankfurt: Iberoamericana/Vervuert. 
